\title{
White but Not Black Holes Might Drive the Universe to The Initial Singularity
}

\author{
Ahmad Yousef ${ }^{1}$ \\ ${ }^{1}$ School of Computational Science and Engineering, McMaster University, Hamilton, Ontario, Canada \\ *Correspondence: mohamas2@mcmaster.ca
}

A white hole is a hypothetical region of spacetime which cannot be entered, namely, it is the reverse of a black hole which can only be entered; where matter/energy cannot escape, see reference 1. Worm holes, however, connect black holes to its corresponding white holes; but they are insensible to our conventional detectors, namely, they might reside in extra physical dimensions. The initial singularity seems to be a gravitational singularity of astoundingly infinite density that consisted of all the mass in an extremely finite spacetime, see reference 2 . The aforementioned singularity happened just before the quantum fluctuations, and therefore rapid expansion, namely, the big bang and subsequent inflation. Inflation that eventually creates our present universe.

Dark energy is known to expand our universe in an accelerative way, behavior that is inconsistent with the increasing number of black holes over time. Such behavior explode a big question; whether the dark energy in the universe had been repulsing against the black holes, as protective action against returning the universe back to a certain singularity. Namely, dark energy might be protecting our universe from massive collapses, by expanding our universe to secure the universe matter/energy from being swallowed by the co-existed black holes. Detailly speaking, without dark energy, our universe might be travelling towards a certain singularity. There are a few theorists think that there is no initial singularity, and the big bang itself is a white hole, see reference 5 . We, however, see the universe in a so different way that simply connects many of the theories together. We hypothesis that the initial singularity has a driver that initiates mass, energy, and spacetime, and that driver inhibits the singularity return. We call this driver, the natural driver, ND. Namely, the aforementioned driver exerts greater dark energy in a proportion way to the number of black holes and their radiuses of event horizon. Once the number of black holes approaches its maximum, white holes will start to emerge in the universe, until their number approaches its maximum in an extremely small spacetime window, and therefore the return of the initial singularity and the emerge of another big bang. The

\section{Spacetime Visible Parachute central infographic} Residence of Dark Energy explains our (extra physical 'invisible' dimensions) hypothesis, reasonizes the present accelerated expansion of the universe, and predicts when the accelerated compression might start. Namely, we predict stoppage in the expansion High density of White Holes synchronized with the first emerge of a white hole. Our model Accelerated theorizes that each black hole is Compression coupled with a white hole, namely, the first white hole might carry all of the information of its corresponding 'first emerged' black hole; and this astoundingly means, the emerge of the first white hole will allow the humanity to read information from very distant past. In that case, the information will travel way faster than light. After approaching the maximum density of white holes, eventually, the natural driver, will return the entire universe back to its initial singularity. Outside the scope of the current project, because the next half of the universe, if happen, might include white holes but not black holes, such hypothesis might propose that the human's retina, could easily detect 'tachyons' due to their expected high energy, unlike nowadays, see reference 6 .

Essential Notification

We think that the white hole center is nothing but a blackhole singularity point; the initial singularity (the biggest white hole), however, might be due to extremely large number of blackholes. Transactional References

[1] Carroll, Sean M. (2004). Spacetime and Geometry.

[2] Hawking, Stephen (2014). The Beginning of Time.

[3] Paál, et al. (1992). Inflation and compactification from galaxy redshifts?

[4] Schmidt and Riess. The Nobel Prize in Physics 2011". Nobel Foundation

[5] Popławski (2012). "Nonsingular, big-bounce cosmology from spinor-torsion coupling". [6] Yousef, A. (2019). "Tachyons but Not Photons Might Generate Conscious Dreams." 